\title{
La pathologie vétérinaire en Guyane française Les affections des bovidés
}

\author{
par H. FLOCH
}

Pendant plus de 10 ans nous avons pu nous intéresser particulièrement à la pathologie animale du fait que nous étions chef du Service vétérinaire et qu'ainsi nous avons pu diriger sur ce sujet intéressant une partie de l'activité de l'Institut Pasteur de la Guyane.

Nous avons pu regrouper nos divers travaux concernant la pathologie des volailles (1) (2) et celle des lapins (3). Nous allons maintenant résumer les constatations que nous avons faites depuis 1939 sur la pathologie des bovins (et bubalins) dans notre département sud-américain.

\section{$*^{*} *$}

Nous avons fait remarquer, dans nos rapports du Service vétérinaire, que si le cheptel bovin guyanais était devenu très peu important (3.000 têtes environ) après l'avoir été bien plus autrefois, il nous paraissait que les affections épizootiques n'étaient pas au premier rang des conditions défavorables ayant abouti à cette diminution, mais que c'étaient bien plutôt les conditions économiques (très opposées au développement de l'élevage local) qui tenaient cette place peu enviable. Un exemple nous a toujours frappé : la Société guyanaise d'élevage, avant la dernière guerre, était uniquement intéressée par l'importation des bovidés brésiliens (quelques années plus tôt, vénézuéliens) et pas du tout par l'élevage local.

La principale région où cet élevage existait encore pratiquement, avant guerre, sur une certaine échelle, était celle de la "Route coloniale no 1 " (Macouria, Kourou, Malmanoury, Sinnamary, Corossony, Iracoubo) où se trouvent des parcours d'élevage de quelque étendue et quelque qualité.

Malheureusement il n'y avait, avant 1939, aucun moyen pour transporter les boufs de cette région à Cayenne, seul centre de consommation important; comme le lait n'était pas, lui non plus, écoulé au chef-lieu (il n'était même pas pratiquement recueilli sauf celui de quelques vaches dans la banlieue de Cayenne) et qu'il n'était pas question de beurre, on comprend fort bien que les campagnards guyanais ne pouvaient attacher un grand intérêt à l'élevage !
Les « bêtes du pays " n'étaient abattues, en réalité, que pour les besoins (très restreints) des bourgs producteurs.

La preuve que ces conditions économiques étaient fort défavorables et qu'il s'agissait bien là de la condition primordiale maintenant l'élevage à un niveau trop peu élevé, est ce qui se passa durant la dernière guerre. Il fallut bien alors songer sérieusement au cheptel bovin (et bubalin) local et y prelever un peu de viande fraîche. On craignit à ce moment d'arriver assez vite à la destruction entière du troupeau guyanais; aussi éleva-t-on des difficultés administratives (souvent et facilement tournées) pour réfréner ces abattages du bétail guyanais. Mais le résultat enregistré (nous l'avions prévu) fut en vérité bien différent de celui auquel certains voulaient $s$ 'attendre : les éleveurs, pouvant enfin écouler leurs produits à bon prix, s'intéressèrent à leurs troupeaux et si, après la guerre, le cheptel était effectivement diminué, il ne l'était que de quelques centaines de têtes et comportait de nombreux veaux, indice d'un élevage rajeuni et "vivant i»; on ne trouvait plus ce que nous avions vu avant la guerre dans certains troupeaux : à peu près autant de taureaux que de vaches!

Soulignons d'ailleurs que la viande des "bêtes du pays » abattues était bien plus belle que celle des bovidés importés péniblement et dans de très mauvaises conditions, du Brésil, dans d'affreuses " tapouilles $»$, comme nous n'avons pas manqué de le souligner durant des années dans nos rapports vétérinaires..., sans grand succès évidemment car, pendant la guerre, il fallait d'abord subsister et tout apport de viande fraiche était le bienvenu.

Ceci prouvait bien, en tous cas, qu'il y avait des possibilités réelles de relever le troupeau guyanais en lui donnant des débouchés normaux, toutes questions de pathologie et d'alimentation restant par ailleurs inchangées.

Avant de passer à la pathologie vétérinaire telle qu'on l'entend habituellement, rappelons que les " savanes » guyanaises ne sont pas, hélas, d'une 
richesse anologuc à la pampa argentine ou aux prairies bretonnes et normandes. Les animaux ne doivent pas être trop nombreux dans cet élevage en liberté qui est le leur habituellement; nous avons pu constater, dans la région du Maroni, il y a plusieurs amnées, un troupeau qui périclitait uniquement par manque de nourriture (carence alimentaire globale).

Nous avons aussi assisté à quelques essais malheureux d'importation et d'acclimatation d'animaux. de races européennes améliorées. Ces animaux, mal nourris, s'adaptaient fort mal et les essais en question se soldèrent par des échecs, suite de carences minérales et azotées. Il ne pouvait en être autrement. En outre, ces essais pêchaient gravement en certains points : c'est ainsi, par exemple, que nous avons vu une fois un éleveur donner fièrement de la farine impropre à la consommation humaine, en remplacement de tourteaux, à des bêtes importées à grand trais !

Le troupeau guyanais peut et doit être amélioré grâce à un choix bien pensé de géniteurs, visant à l'amélioration des qualités de boucherie et des qualités laitières, mais, d'autre part; il est capital de se rappeler quo lcs bovins possédant du sang zébu assez riche résistent ici toujours mieux que les autres aux affections locales, notamment à la trypanosomiase à $T$. viennei ; ils résistent, certainement aussi, bien mieux au climat et aux autres conditions d'existence de ces animaux en Guyane. Deux croisements seraient donc logiquement à mener de front pour améliorer les qualités productrices du cheptel guyanais tout en conservant son accoutumance au milieu local.

\section{$* * *$}

C'est en 1918 que Léger et Vienne décrivirent sous le nom de T. guyanense (appelé plus tard T. viennei) un trypanosome causant une trypanosomiase bovino mortcllo à Rémire-Montjoly (4). Ensuite, jusqu'à notre arrivée en 1938, ce flagellé n'a été retrouvé qu'une fois, à Rémire, par Berny et Saint-Prix, en 1935. Cette trypanosomiase bovine pouvait donc paraître exceptionnelle en Guyane française. En réalité T, viennei n'y était pas suffisamment recherché.

En effet, de 1940 à 1942, nous l'avons décelé 12 fois (à l'abattoir de Cayenne) chez des bovins brésiliens ou guyanais. Les régions de Rémire, de Cayenne (Montabo), de Macouria, de Sinnamary et de 'l'rou-Poisson, en ces années, ont été trouvées atteintes par la trypanosomiase bovine qui intéresse en réalité tout le département, comme nous l'avons confirmé depuis, car, pratiquement, chaque année depuis lors, nous rencontrons $T$. viennei.
Signalons qu'en 1946 nous l'avons trouvé chez un buffle du Jardin botanique à Cayenne.

On retrouve en Guyane française les trois formes cliniques de l'affection (forme subaiguë, forme aiguë, et forme chronique), décrite en Afrique sous le nom de « souma ». Les symptômes observés ne sont d'ailleurs pas rapportés, par les éleveurs, à la trypanosomiase; pour eux l'animal « maigrit », a "de la diarrhée » ou « est tombé » et ceci parce que, par exemple, son alimentation est insuffisante, ou qu'il est anémié à la suite des piqûres de taons. Notons déjà cependant ce dernier fait d'observation qui établit une relation de cause à effet entre une maladie mortelle des bovidés et les tabanidés.

La trypanosomiase bovine à $T$. viénnei nous paraît la plus importante, actuellement, des causes de morbidité et de mortalité des bovidés en Guyane française.

La trypanosomiase à $T$. viennei a été rencontrée en Guadeloupe (où le trypanosome aurait été introduit par « du bétail sénégalais » vers 1875) (5); au Venezuela (où elle serait apparue « quelques années avant 1920 » d'après Tejera) (6) à la suite d'importation de zébus africains; à la Martinique (où $T$, viennei aurait été introduit par du bétail vénézuélien et colombien en 1027) (7) et au Brésil, comme nous l'avons constaté, où 'elle'a dû être introduite, pensons-nous, par des zébus d'origine africaine.

\section{** $*$}

T. viennei est en général considéré comme iclentique à T. vivax $=T$. cazalboui (Roubaud, Hoare et Brown).

Signalons cependant que F. Mesnil avait 'trouvé quelques différences biologiques entre T. cazalboui (décrit en 1904 par Cazalbou dans le Haut-Niger) et T. viennei; le premier est naturellement très pathogène pour les équidés en Afrique, tandis qu'aucune infection naturelle authentique de ces animaux par le second n'a été observée. T. cazalboui est, expérimentalement aussi, bien plus pathogène (pour le cheval, le mouton et le lapin) que ne l'est $T$. viennei. Enfin celui-ci n'est pas apte à évoluer expérimentalement chez les glossines' vecteurs spécifiques de T. cazalboui (8).

Toujours est-il qu'en Afrique, à côté du T. vivax typique, on a décrit deux trypanosomes voisins: T. caprae, plus massif, causant une trypanosomiase guérissant spontanément et $T$. uniforme plus petit. Hoare et Brown séparent T. uniforme de $T$. vivax par la recherche de la longueur moyenne sur 10 exemplaires; si cette longueur est moindre que $18 \mu$ il s'agit de $T$. uniforme, si elle dépasse $20 \%$ il s'agit de T. vivax, 
En pratiquant cette « biométrie » nous avons trouvé en Guyane un grand (longueur moyenne $22 \mu$ ) et un petit $T$. viennei (longueur moyenne $16 \mu .5$ ). Le premier pourrait donc correspondre au $T$. vivax et le second au $T$. uniforme africain.

Ajoutons que, classiquement, T. vivax est doué d'une mobilité remarquable et que $T$. uniforme est bien moins rapide; or Tejera, qui a rencontré un trypanosome du type viennei au Venezuela, écrit que ses parasites « agitaient les globules rouges mais jamais ne traversaient le champ du microscope " ce qui semble bien être plus caractéristique d'un T. uniforme que d'un vivax véritable.

\section{$*^{*} *$}

L'insecte vecteur de T. viennei en Amérique était inconnu jusqu'à nos études.

T. vivax est transmis en Afrique par les tsé-tsés, qui n'existent pas, on le sait, sur le Nouveau Continent, mais il y est admis que, lorsqu'un foyer de trypanosomiase est constitué, des insectes piqueurs plus ou moins banaux (les stomoxes par exemple) peuvent transmettre la maladie.

Le trypanosome effectue son cycle de développement chez l'invertébré uniquement dans la trompe; ce fait aurait pu faciliter son adaptation à d'autres insectes que son vecteur de choix; de là probablement sa fixation définitive en Amérique tropicale.

Fin 1953 nous avons observé un foyer important (7 troupeaux atteints) de trypanosomiase bovine à Montjoly, commune proche de Cayenne, ce qui nous a permis de suivre de plus près l'évolution et de reconnaitre les particularités suivantes de l'affection :

a) les poussées épidémiques coïncident en général avec la fin de la saison sèche et des invasions de taons :

b) ces bouffées épidémiques s'atténuent et disparaissent avec les tabanidés lors des premières pluies importantes.

Fn outre nous avons alors recueilli deux especes de taons (T. importunus et $T$. sp.); les dissections de ces insectes nous ont permis de déceler :

a) des formes trypanosomiennes (forme des vertébrés) dans le sang ingéré par les insectes;

b) des formes leptomonas dans les trompes de T. importunus, qui est donc un vecteur de $T$. viennei sur le continent américain.

En effet, si les dissections de 60 exemplaires de notre $T$. sp. ne nous ont pas permis d'y déceler des flagellés (trompe et intestin), sur $140 \mathrm{~T}$. importunus, nous avons trouvé trois fois des formes crithidia en colonies dans le labre et une fois un $T$. viennei de forme sanguicole typique dans l'estomac d'un insecte qui venait de se gorger.
La coexistence de l'épizootie et de la présence des flagellés dans le labre de T. importunus nous permet d'admettre que $T$. vivax a pu trouver en Guyane française, au moins en cette espèce de Tabanidés, un véritable hôte de remplacement. Nous n'avons malheureusement pas pu tenter d'infections expérimentales (9).

\section{$*^{*} *$}

La faune tabanide est très riche en Guyane. Nous avons pu enregistrer dans notre département sudaméricain la présence des espèces suivantes : Chrysops variegatum (de Geer), Diachlorus bicinctus (Fabricius), Dichelacera capricornis, Dichelacera januarii (Wiedemann), Elaphella arvus (Wiedemann) Fidena pseudoaurimaculatus, Fidena sp., Lepiselaga crassipes (Wiedemann), Tabanus cayennensis (Fabricius), Tabanus importunus (Wiedemann) $(=T$. albidicollis Macquart).

Les espèces suivantes ont été en outre rapportées par Surcouf : Diachlorus scutellatus Macquart, Dichelacera marginata Macquart, Tabanus angustifons Macquart, Tabanus casteneus Macquart, Tabanus füllax Macquart, Tabanus flavibarbis Macquart, Tabanus fulvilateralis Macquart, Tabanus guyanensis Macquart, Tabanus impressus Wiedemann, Tabanus ochraceus Macquart, I'abanus pulverulentus Bigot, Tabanus rubidus Macquart, Tabanus rubripes, Chrysops tristis Fabricius.

Au point de vue de la thérapeutique de la trypanosomiase à $T$. vivax signalons l'interêt de la Trypadine (Chlorure de Dimidium ou, mieux, Chlorure de diamino-2-7 phényl 9 méthyl 10-phénanthridinium) (*).

On utilise par la voie veineuse des solutions en eau distillée, à 1 ou $2 \%$, aux doses de 1 à $2 \mathrm{mg}$ par kilogramme de poids vif : une injection en général suffit mais on peut être amené à en pratiquer deux. On peut utiliser à la rigueur la voie intramusculaire mais on observe assez souvent ainsi quelques phénomenes réactionnels locaux.

En chimiothérapie préventive (prophylaxie chimique) on utilise la même solution à doses un peu plus faibles : 0,80 à $\mathrm{l} \mathrm{mg}$ par kilogramme. Les animaux résistent alors environ un mois à la contagion. Lorsqu'un cas de trypanosomiase à $T$. viennei est signalé dans un troupeau, tout celui-ci est à traiter immédiatement. On peut même envisager un traitement preventif à la fin de la saison sèche lorsque, nous l'avons vu, la maladie se manifeste au maximum.

(*) Specia, Paris. 
Le seul incident signalé est une photosensibilisation (survenant surtout chez les animaux à robe claire) qui apparaît quelques semaines après l'injection (doses trop fortes ? trop grande exposition des animaux à la lumière ? insuffisance de l'alimentation et de l'abreuvement ?). Traitement : mettre le bétail à l'ombre dans la journée pendant une semaine (10).

Un autre trypanocide l'Antrycide (**) est aussi rccommandablo; ce produit parait même faire preuve d'une supériorité certaine sur la Trypadine.

\section{$*^{*}{ }^{*}$}

Bien plus rarement que $T$. viennei nous avons trouvé chez des boeufs brésiliens et guyanais, un très grand trypanosome du type theileri classique; la longueur moyenne, recherchée sur 10 individus, était de $51 \mu$ dont 23,5 pour le flagelle.

T. theileri est consideré, en général, comme non pathogène. Il est difficile de le déceler par simple examen coloré de sang; car il est très rare dans la circulation périphérique. La méthode des cultures donne des résultats bien meilleurs. Quelquefois, cependant, ont été signalées des infections intenses; ces cas semblent, toutefois, être extrêmement rares, si rares qu'on les publie.

T. theileri serait souvent un verritable « virus de sortie 》 se greffant sur une infection primitive : peste bovine, piroplasmose, anaplasmose, autres trypanosomiases animales.

Sa constatation dans un troupeau guyanais doit donc, surtout, éveiller l'attention au point de vue de l'existence probable de la trypanosomiase à $T$. vien$n e i$ dans ce troupeau. Nous avons effectivement eu l'occasion de constater un cas d'infection intense par T. theileri et il y avait alors coexistence chez le même animal d'une infection à $T$. vivax typique.

T. theileri se présente en Guyane sous sa forme habituelle et sous une petite forme dont la longueur moyenne, sur dix spécimens, est seulement de 34 . dont 18,5 pour le flagelle.

Ajoutons que T, theileri est cosmopolite. En Amérique du Sud, il a été signalé en Uruguay (Peter, 1910), au Brésil (dans l'État de Saô-Paulo, Carini, 1911), en République Argentine (Dias et Zuccarini, 1924).

$$
* * \text { * }
$$

Une épizootie a sévi en mai 1940, sur des bovidés de provenance brésilienne qui avaient ćtć placés à Crique Anguille dans la même étable que des bêtes du cheptel autochtone et qui furent attaqués dès leur arrivée, par de nombreux Boophilus annulatus microplus (11).

(**) Imperial Chemical Limited, Wilmslow, Manchester.
Les animaux malados ne mangeaient plus, s'isolaient, perdaient leurs poils et maigrissaient très rapidement.

A l'autopsie', on constata que la rate était hypertrophiée et très congestionnée, que le foie, d'aspect extérieur normal, était jaunâtre à la coupe, que le cœur était mou et que poumons et reins étaient congestionnés.

On no put isoler de germes microbiens pathogènes, mais on décela d'assez nombreux piroplasmes dans le sang; il s'agissait de P. bigeminum.

La durée de la période d'incubation prouvait bien que les animaux avaient été contaminés par un piroplasme d'origine animale guyanaise transmis par des tiques du genre Boophilus; que nous avons elles aussi trouvé infectées : dans leur liquide coxal nous avons observé de nombreuses formes parasitaires allongées, caractéristiques, qui nous ont permis de poser, aussi facilement que l'examen du sang des boeufs, le: diagnostic de piroplasmose.

$$
*^{*} *
$$

Durant de nombreuses années nous avons étudié la question des brucelloses animales dans notre département sud-américain.

En 1941, sur 1.139 séro-diagnostics pratiqués chez des bovins du Brésil, 113 soit $15 \%$ d'entre eux ont été trouvés positifs. Les particularités de groupement de ces résultats positifs, en relation avec les différentes régions d'origine, prouvent que certains troupeaux de l'État de Para, au Brésil, sont infectés par B. abortus bovis. Par contre, cette même année 20 séro-diagnostics, chez des bovidés guyanais, ont été négatifs.

En 1942, 595 séro-diagnostics à l'aide de' la souche B. abortus suis' $S, 600$ ont été aussi pratiqués sur des sérums de bovidés provenant de l'abattoir de Cayenne; 81 d'entre eux (13\%), dont quelques-uns d'origine guyanaise, agglutinaient la suspension microbienne au taux minimum de $1 / 50$, considéré comme positif.

Les années suivantes, les chiffres correspondants ont été : 1943, 204 sérums examinés, 26 positifs $(13 \%) ; 1944,208$ sérums examinés, '25 positifs $(12 \%) ; 1945,63$ sérums examinés, 9 positifs $(14 \%)$.

Le pourcentage de résultats positifs est donc remarquablement constant, puisque nous avons trouvé $12 \%$ en $1944,13 \%$ cn 1942 et en $1913,14 \%$ on 1915 . $15 \%$ en 1951 , et en six ans, sur 2.230 sérums, $14 \%$.

Quelques avortements suspects ayant été observés durant les années antérieures, Savane Matiti, une enquête fut effectuée en 1945 : les examens pratiqués sur des vaches ayant avorté précédemment et sur des vaches saines ont été négatifs (trois hémo-cultures, trois lacto-cultures, trois séro-diagnostics, deux 
séro-lacto-diagnostics); un seul séro-lacto-diagnostic, chez une vache apparemment saine, fut légèrement positif (à 1/50).

L'affection n'a manifestement pas, actuellement en Guyane, une grande importance, mais elle pourrait peut-être l'avoir lorsque notre élevage cessera d'être embryonnaire, car elle est certainement bien plus répandue dans les états voisins du Brésil (12).

Il n'est pas inutile d'ajouter que lorsque nous avons enfin isolé par hémo-culture une Brucella d'origine humaine ce fut (à notre étonnement) une souche de Brucella melitensis (13).

Le charbon bactéridien a sévi autrefois en Guyane. En mai 1927, une épizootie importante (plus d'une centaine de bovidés périrent alors à Cayenne) se manifesta chez des animaux importés du Brésil : d'autres cas, l'année suivante, furent diagnostiqués à Matiti sur des bêtes du cheptel local.

Depuis lors, la vaccination systématique annuelle du troupeau guyanais est pratiquée ef nous n'avons. pour notre part, jamais rencontré de charbon bactéridien de 1939 à 1954.

Devant l'impossibilité de recevoir le vaçcin habituellement employé en Guyane, l'Institut Pasteur de Cayenne a étudié et mis au point, pendant la guerre, à partir d'une souche de bactéridie charbonneuse dont l'atténuation correspond à celle du premier vaccin de Pasteur, un vaccin-spores liquide gélosé et aluné (G. Ramon et $A$. Staub) qui est employé depuis lors avec succes pour la totalité du cheptel bovin et bubalin guyanais. Il est aussi utilisable chez les autres espèces domestiques sensibles.

Après un an de conservation à la température du laboratoire à l'abri de la lumière, les spores contenues dans les ampoules de vaccin ont encore toute leur vitalité, ce qui permet une marge d'utilisation possible intéressante.

L'obtention de cultures de Bactéridie charbonneuse, riches en spores, n'est pas sans relation avec les milieux nutritifs employés. Cette question nous a été particulièrement intéressante à étudier lors de la mise au point de notre vaccin-spores.

J. Bordet et E. Renaux (14) ont montré qu'une variante obtenue à partir de cultures classiques de charbon était sporogène (colonies plates, à surface humide, présentant des expansions et blanchissant rapidement), tandis qu'une autre variante (vers laquelle tendent les souches entretenues au laboratoire sur gélose ordinaire) est dite asporogène (colonies plus régulières, plus épaisses, plus ternes). L'apparition de cette dernière variété est nettement favorisée par le calcium. comme le montrent les cultures comparées en gélose calcifiée et en gélose oxalatée.
Notre souche vaccinale de $B$, anthracis nous a permis de constater ces variantes, mais une bien plus faible dose d'oxalate sodique que celle préconisée (gélose oxalatée à $2 \%$ ) par Bordet et Renaux nous permet régulièrement d'obtenir et de conserver la variante sporogène; ceci tient évidemment au fait que le calcium est presque totalement absent des eaux de Cayenne. Une dose trop élevée d'oxalate nous a rnême paru défavorable à la production de spores.

\section{** $*$}

Nous n'avons diagnostiqué qu'une fois, en 1939, le charbon symptomatique, à l'I. P. de la Guyane.

\section{$*^{*} *$}

Par quatre fois nous avons isolé au laboratoire une salmonelle bovine : Salmonella bovis morbificans dont voici les principaux caractères biochimiques et sérologiques : glucose + gaz ; lactose - ; maltose + ; saccharose - ; mannite + ; dulcite + ; sorbite + ; glycérine + ; amidon - ; lait - ; M.R. - ; indol -; agglutination par sérum antisalmonelles ++ .

Ces salmonelles ont été isolées de zones jaunâtres de nécrose, superficielles en général, de petites dimensions (celles de pièces de $0 \mathrm{fr}$. 50 et 1 franc), de poumon et de foie, mais aussi de ganglions, de bovidés abattus en bonne santé apparente.

$S$. bovis morbificans a quelquefois été isolé de gastro-entérites chez l'homme.

Une culture de Chromobacterium violaceum a été obtenue de la sérosité pulmonaire d'un taurillon abattu parce qu'atteint de broncho-pneumonie.

C. violaceum est en général considéré comme un germe saprophyte. Cependant, en ces dernières années surtout, plusieurs cas d'infections subaiguës et mortelles par ce germe, ont été signalés chez l'homme.

Notre souche était irès pathogène expérimentalement pour le cobaye.

Le groupe des « bacilles violets » est bien hétérogène et les propriétés attribuées à son pigment violet, qui serait un pigment respiratoire, varient beaucoup suivant les auteurs; celui de notre souche était soluble dans l'alcool éthylique et dans l'éther (partiellement), insoluble dans l'eau, la benzine et le chloroforme; la solution alcoolique virait au vert bleu par addition d'ammoniaque.

L'aspect des colonies isolées sur gélose ordinaire et l'action hémolytique sur gélose-sang, permettaient de constater plusieurs « variantes ». 
Nous étendons le pouvoir. pathogène spontané, réel pour divers auteurs, de C. violaceum, à d'autres germes de l'eau et du sol (genres Chromobacter. Achromobacter, Pseudomonas, notamment). Pour nous il est certain que ces bacilles peuvent devenir pathogènes (septicémies, localisations diverses notamment broncho-pulmonaires) dans certaines conditions lorsqu'ils sont absorbés par les animaux domestiques en grandes 'quantités, avec l'eau des mares ou les fourrages très pollués.

\section{***}

La fièvre aphteuse se manifeste de temps en temps en Guyane française mais, en général, fort heureusement de façon bénigne.

En septembre 1942, c'est le troupeau de buffles de l'Administration pénitenciaire à Saint-Laurent qui est atteint; le mois suivant l'épizontie touche les troupeaux bovins de la commune.

En mars-avril 1946 plusieurs troupeaux bovins et bubalins furent atteints dans la région de Cayenne.

Depuis jusqu'en avril-mai 1954 nous ne rencontrâmes plus de fièvre aphteuse en Guyane mais à ce moment la maladie réapparut sur un troupeau de bovins de boucherie importé de Surinam où la fièvre, aphteuse était alors reconnue et signalée.

I'affection put être circonscrite au troupeau en question et ne's'étendit pas au cheptel guyanais.

\section{$*^{*} *$}

Un cas de paraplégie des bovidés a été observé et étudié en $1942^{\circ}$.

L'animal atteint ćtait frćquomment mordu par dos vampires. $A$ la suite de ces morsures des cas de rage ont été signalés, on le sait, dans les pays avoisinants (Brésil, T'rinidad, Guyane anglaise, notamment).

Nous avóns recherché sans succès dans le système nerveux du bovidé en question les.corps de Négri et les inoculations pratiquées sur cobaye sont restées négatives.

Il ne s'agissait donc pas de rage paralytique mais il faut toujours penser en Guyane française à la possibilité de l'apparition de cette affection.$$
*^{*} *
$$

En 1940 (15) nous avons signalé la présence en Guyane, chez des bovidés importés, mais aussi dans le troupeau autochtone, de l'affection dénomnriée " Peste dos Polmoës' " par les auteurs brésiliens.

Il s'agit d'une maladie multiforme à synonymie très variée: : dysenterie ou diarrhée des veaux », ' "pneumoentérites' », « abcès périarticulaires 》 etc., cue à un virus filtrant ( $O$. de Magalhaès) favorisant la sortie de nombreux germes et notamment de Pasteurella.

Le pronostic est toujours mauvais, sauf dans les cas chroniques; la mortalité peut atteindre 99 à $100 \%$ des cas pendant les épizooties ; entre celles-ci elle peut ne pas dépasser $10 \%$. Magalhaès préconise en thérapeutique un sérum préparé à partir de l'uitra-virus.

La maladie est probablement transmise par les tiques; surtout par Boophilus annulatus microplus très fréquent en Guyane.

Prophylactiquement il faut isoler les animaux malades et détruire leurs tiques; Magalhaès propose en outre un vaccin préventif spécifique.

\section{***}

Parmi 28 microfilaires animales que nous avons décrites en Guyane française (16) nous en rencontrâmes deux du bceuf et une du buffle.

La première microfilaire des bovidés guyanais n'a pas de gaine et mesure de 160 à $190 \mu$ de long sur 7 à $10 \mu$ de large; la seconde est entouréel d'une gaine assez bien colorable, étroite mais longue.

Chez les bovidés importés du Brésil nous n'avons trouvé, jusqu'ici, que la seconde de ces microfilaires.

La microfilaire du buffle, en Guyane, est sans gaine: fixée en attitude flexueuse et mesure 188 à $233 \mu$ de long sur 4,5 à 6 u. de large.

\section{***}

Fréquemment à l'abattoir des saisies de poumons de bovidés sont effectuées en raison de strongylose bronchique (Dictyocaulus viviparus). Institut Pasteur de la Guyane française.

\section{BIBLIOGRAPHIE}

(1) H. FLOCH. - Sur la pathologie vétérinaire en Guyane française. Les affections des volailles. 10 Salmonellose. Archives de I'Institut Pasteur de la Guyane française. Publication no 293, aồt 1953

(2) $\mathrm{H}$. FLOCH. - Sur la pathologie vétérinaire en Guyane française. Les affections des volailles. $2^{\circ}$ Pasteurellose, Coccidiose, Variolo-diphtérie, Syngamose, Spirochétose, Parasitismes divers, Tumeurs. Archives de l'Institut Pașteur de la Guyane. Publication no 295, septembre 1953.

(3) $\mathrm{H}$. FLOCH', - Sur la pathologie vétérinaire en Guyane française. Les maladies du clapier. Archives de l'Institut Pasteur de la Guyane française. Publication no 296, septembre 1953. 
(4) M. LÉGER et M. VIENNE. - Épizootie à trypanosomes chez les bovidés de la Guyane française. Bull. Soc. Path. Exot., t. XII, novembre 1919 pp. 258-266.

(5) H. FABRE et M. BERNARD. - Sur un nouveau foyer de trypanosomiase bovine observé à la Guadeloupe. Bull. Soc. Path. Exot., 1926, p. 435.

(6) E. TEJERA. - Trypanosomiases animales an Vénézuéla. Bull. Soc. Path. Exot, 1920, p. 297.

(7) M. CAROUGEAU. - Trypanosomiase bovine à la Martinique. Bull. Soc. Path. Exot., 1929, p. 247 .

(8) E. ROUBAUD, J. COLAS-BELCOUR et H. GASHEN, - Le trypanosome des Antilles. Trypanosoma viennei a-t-il perdu l'aptitude à évoluer chez les glossines? Bull. Soc. Path. Exot, 1938, p. 374.

(9) H. FLOCH et P. de LAJUDIE. - Sur la transmission naturelle du Trypanosomia viennei Lavier 1921. Archives de l'Institut Pasteur de la Guyane française. Publication no 79 , juin 1944.

(10) F. DEBEKER. - Observations sur les accidents toxiques survenus à la suite de traitements de la trypanosomiase bovine par le bromure de Dimidium dans quelques troupeaux du Kuango. Bulletin Agricole du Congo belge, vol. III, no 1, février 1954, p. 225.
(11) H. FLOCH et E. ABONNENC. - Piroplasma bigeminum et Boophilus microplus en Guyane française. Archives de l'Institut Pasteur de la Guyane française. Publication $n^{\circ} 7$, février 1953.

(12) H. FLOCH et P. DE LAJUDIE.- Sur les brucelloses en Guyane française. Archives de l'Institut Pasteur de la Guyane française. Publication $n^{\circ} 18$, août 1941 .

(13) H. FLOCH. - Étude d'une souche de B. melitensis isolée par hémoculture à Cayenne. Archives de l'Institut Pasteur de la Guyane française. Publication no 39, avril 1942.

(14) J. BORDET et E. RENAUX. - L'Influence du calcium sur l'évolution des cultures de charbon. Annales de l'Institut Pasteur, juillet 1930, t. XLV, p. 1.

(15) H. FLOCH. - Une maladie des bovidés à connaitre en Guyane : La « Peste dos Polmoës " des auteurs brésiliens. Archives de l'Institut Pasteur de la Guyane française. Publication $\mathrm{n}^{\circ} 14$, juin 1941.

(16) H. FLOCH et DE LAJUDIE. - Sur vingt-huit microfilaires animales en Guyane française. Archives de l'Institut Pasteur de la Guyane française. Publication no 53, septembre 1942. 